\title{
Successful salvage resection of locoregional colon cancer recurrence. Report of four cases
}

C. Emmanouilides*1, M. Karampola ${ }^{1}$, Ch. Efthymiadis²,

P. Fotiadis'², J. Tzeveleki², G. Basdanis²

'Interbalkan Medical Center, Department of Oncology

2Interbalkan Medical Center, Department of Surgery

\begin{abstract}
Locoregional colorectal cancer recurrence after curative resection remains a clinical problem despite improvements in surgical techniques. A surgical approach is usually preferred, although controversies exist. Retrospective studies have identified risk factors that may predict successful outcomes after reoperation. In the current manuscript , the experience of our tertiary oncology center involving cases with complicated locoregional recurrences is reported. Four such patients were identified, age 55-69 years old, who were treated with an aggressive surgical intervention in the setting of multimodality treatment including postoperative chemotherapy and radiation. Despite the presence of risk factors, such as involvement of adjacent structures, an excellent disease-free survival was achieved. We, therefore, propose that aggressive surgical approach supported by modern chemotherapy and occasionally radiotherapy for the treatment of colorectal cancer locoregional relapses should be considered in the setting of a multidisciplinary oncologic evaluation.

Keywords: Colon cancer • Retroperitoneal relapse • Surgery

(c) De Gruyter Open
\end{abstract}

\section{Introduction}

Early-stage colorectal cancer is usually curable by surgical resection. However, approximately $30 \%$ of patients relapse, most commonly with hepatic metastasis. In a subset of patients, locoregional recurrence may be the only site of relapsed disease. Locoregional colon cancer recurrence is a relatively uncommon event, occurring in less than $10 \%$ of operated patients. The most common type of local recurrence is anastomotic that is commonly managed with repeat resection, usually with good results. Nodal or retroperitoneal recurrences may be more problematic, as they may involve adjacent organs and structures. There are no universally accepted guidelines for the management of such patients. Nevertheless, a surgical salvage approach is preferred when complete resection is deemed feasible. The intended goal of the resection is to offer a second chance for cure. If such an aggressively treated patient further relapses, it may be conceded that at least local tumor control and prolongation of disease-free survival was offered. Accordingly, certain authors [1] propose an aggressive approach, whereas others reserve surgical salvage for a select group of low-risk patients [2].

We hereby report the outcome of four patients followed at the medical oncology clinic of Interbalkan Hospital, who experienced locoregional recurrence of colon cancer, initially treated with curative surgery. All the four patients were referred to an experienced oncologic surgeon for salvage resection.

\section{Materials and methods}

The medical records of the medical oncology ward of Interbalkan Medical Center for the period 2010-2013 were retrospectively reviewed. Eighty-three patients, who had received adjuvant chemotherapy for colorectal 
cancer, were identified. Four of these patients with regional relapse were identified and are presented below.

Initial selection for aggressive surgical management of locoregional relapse was based on routine computerized tomography (CT) imaging. If patients fulfilled the criteria of lack of distal metastasis, the extent of the disease was further assessed with magnetic resonance imaging (MRI) of the abdominal and retroperitoneal areas. All patients had to have undergone colonoscopy within 6 months prior to surgery; otherwise, it was performed preoperatively.

\section{Case 1}

The patient, GG, is a 55-year-old male patient who underwent a right hemicolectomy in May 2010 for a T4, N0 poorly differentiated adenocarcinoma of the ascending colon. Postoperatively, he received adjuvant chemotherapy with oxaliplatin and capecitabine for 5 months.

Upon reevaluation, in December 2010, he was found to have retroperitoneal relapse, at the site of the initial resection. There was no evidence of distal metastasis. CT imaging revealed a 4-cm irregular mass involving the psoas muscle. His carcinoembryonic antigen (CEA) was within normal limits. He underwent a repeat laparotomy. The tumor involved the retroperitoneal area anterior to the iliac vessels, the area of the ileocecal anastomosis as well as the adjacent small bowel loops. The involved area was completely resected en bloc. The patient recovered uneventfully.

The resection was deemed complete. The tumor did not harbor K-ras mutation. Postoperatively, he received chemotherapy treatment with irinotecan, leucovorin, 5-fluorouracil (FOLFIRI) and cetuximab with good tolerance. In May 2011, he received radiation therapy to the involved right lateral abdominal area at the dose of 4860 cGy. Subsequently, he continued the same chemotherapy treatment until November 2012, when treatment was stopped altogether. He remains on close follow-up and without evidence of disease, as of June 2014.

\section{Case 2}

The patient, OM, is a 58-year-old male patient who underwent a sigmoidectomy in 2008 for a stage III moderately differentiated adenocarcinoma. He received adjuvant oxaliplatin and capecitabine for 6 months (XELOX). Two years later, he developed a large midline lower abdominal retroperitoneal mass. At that time, his CEA was normal and performance status was good. There was no evidence of distal metastasis.

After multidisciplinary consultation, he was deemed operable and underwent an extensive resection of several retroperitoneal tumors, the largest measuring $6 \mathrm{~cm}$, consistent with relapse of his adenocarcinoma. The resection was complete. The patient recovered uneventfully. His tumor was positive for K-ras mutation. Postoperatively, he received irinotecan, leucovorin, 5-fluorouracil (FOLFIRI) with bevacizumab for six months. He remains disease-free at 40 months since his last operation.

\section{Case 3}

The patient, LS, is a 64-year-old male who underwent a resection of a supposedly T3, N0 sigmoid adenocarcinoma at another institution in August 2011. He then came to our hospital for consideration for adjuvant chemotherapy. Upon evaluation with CT scans, he was found to have a residual $4-\mathrm{cm}$ mass adjacent to the urinary bladder at the tumor bed area. He was reoperated and the residual nodal carcinomatous mass was removed. He then received adjuvant chemotherapy with oxaliplatin, leucovorin and 5-fluorouracil (FOLFOX) for 6 months.

A year later, CT evaluation revealed a right pelvic mass of $5 \mathrm{~cm}$. CEA was normal. Colonoscopy at that time showed tumor at the site of the anastomosis. It was unclear whether this represented a frank de novo cancer or was a result of the inadequate first surgery initially performed at an inexperienced institution. There was no evidence of distal metastasis.

He underwent a surgical exploration with the intention of a complete resection. As the tumor also involved the bladder wall, a urologist assisted during the procedure. Complete resection of the involved bowel, retroperitoneal area, and partial cystectomy were successfully performed. An isolated peritoneal implant of the upper abdomen unexpectedly found during the operation was also removed. Temporary bilateral nephrostomies were placed. The patient required a hospital stay of 8 days. As he had no mutations of $K$-ras, he received postoperative chemotherapy with FOLFIRI and panitumumab for 4 months. He remains 
with no evidence of disease at 23 months since his last surgery.

\section{Case 4}

A 69-year-old female was diagnosed with stage III adenocarcinoma of the ascending colon in December 2010. After resection, she received adjuvant FOLFOX chemotherapy for 10 biweekly cycles. In January 2013, routine imaging evaluation revealed a right pelvic retroperitoneal mass of $4 \mathrm{~cm}$. CEA was elevated at $15 \mathrm{ng} / \mathrm{ml}$. Colonoscopy and further staging were negative. She underwent a complete resection of the retroperitoneal metastasis and recovered uneventfully. Postoperative FOLFIRI was administered for eight cycles. The patient experienced relapse with isolated liver metastasis and subcutaneous nodules in January 2014. She has responded to XELOX-bevacizumab chemotherapy.

\section{Discussion}

Isolated retroperitoneal recurrence of previously resected colorectal cancer is a relatively uncommon and challenging problem, often resulting in patients' demise. Our cases illustrate the feasibility of successful salvage resection, even of complex locoregional colon cancer recurrence, in the setting of multidisciplinary approach within an experienced tertiary center. Although not sufficiently substantiated, an aggressive approach aimed at complete resection is usually adopted. Nevertheless, there is sparse evidence in the published medical literature regarding the merits of such an approach. In fact, a literature review published in 2011 by $\mathrm{Ho}$ et al [3], involved only 110 patients.

Our small series of four patients serve to support the notion that a surgical approach is justified. Two of the four patients presented with considerable tumor bulk, one had involvement of the bladder wall and another had extension to the psoas muscle. These presentations could have possibly deterred an operation elsewhere as they are empirically considered of adverse prognosis.

Resection of the relapse seems to offer long-term benefit to patients according to published studies. Median overall survival in the reported studies ranges between 30-40 months overall. Bowne et al. [4], report a 66-month median survival for the $\mathrm{R} 0$ resection subset of 56 patients. Based on the above, it is not surprising that three out of our four patients have not relapsed.

In all of our patients, a complete $\mathrm{RO}$ resection was achieved. An Ro resection seems to be the most important factor for long-term survival or cure as reported by several studies [4,5]. Other adverse prognostic factors that have been reported include tumor size $>5 \mathrm{~cm}$, age $>60$, relatively elevated CEA, short interval since primary surgery and, importantly, more than one metastatic site and involvement of the mesenteric nodal basin. In the retrospective analysis of 100 patients with locoregional recurrence of colon cancer, having received adjuvant chemotherapy, stage III at presentation and symptomatic disease were identified as adverse prognostic features. The presence of central retroperitoneal recurrence seems to be associated with an ominous prognosis [4]. Such patients should perhaps be considered separately. This was retrospectively analyzed by Dumont et al. reviewing a 20-year database from Institut Gustave Roussy [6]. They identified 31 patients divided in two groups: those who had just lymph node recurrence and those who had a more advanced locoregional recurrence. None of the latter group of eight patients remained disease-free as opposed to $26 \%$ of the lymph node recurrence group.

In spite of the fact that our patients displayed some of those features, they have fared well with no evidence of recurrence so far in three out of four. Clearly, because of the small size of the cohorts presented in the literature, much caution is required when interpreting univariate analysis results of the published studies. In addition, the validity of the proposed risk factors for subsequent relapse is further challenged by the emergence of more active postoperative chemotherapy. It should be noted that our patients were managed not only with resection but also with postoperative chemotherapy and in one case with radiation. It is well known that chemotherapy for colon cancer has significantly improved in the last decade and may have contributed to the successful outcomes, while the patients reported in the retrospective series may have not had optimal chemotherapy according to current standards. Currently, there are no guidelines regarding the duration and the nature of postoperative chemotherapy, nor is there any evidence that the use of monoclonal antibodies is of benefit in patients with no measurable disease. Consequently, our patients received postoperative treatment according to the best 
clinical practice and personal wishes. However, it can be recommended that a new-generation chemotherapy regimen should follow complete surgical resection of the relapsed tumor.

In conclusion, based on our experience as well as the limited published evidence, it is reasonable to attempt surgical approach with curative intent in patients with retroperitoneal or nodal colorectal cancer recurrence.

\section{References}

[1] Stipa S, Nicolanti V, Botti C et al (1991) Local recurrence after curative resection for colorectal cancer: frequency, risk factors and treatment. J Surg Oncol Suppl 2:155-60.

[2] Turk PS, Wanebo HJ (1993) Results of surgical treatment of nonhepatic recurrence of colorectal carcinoma. Cancer 71: 4267-77

[3] Ho TW, Mack LA, Temple WJ (2011) Operative salvage for retroperitoneal nodal recurrence in colorectal cancer: a systematic review. Ann Surg Oncol, 18: 697-703.

[4] Bowne WB, Lee B, Wong WD, Ben-Porat L et al (2005) Operative salvage for locoregional recurrent colon cancer after curative resection: an analysis of 100 cases. Dis Colon Rectum 48:897-909.
Obviously, careful selection of suitable candidates is indicated, taking into account location of the tumor, involvement of vital structures or vessels and comorbidities. Occasionally, vascular surgery or urologic assistance may be required. It is imperative that such patients be evaluated by multidisciplinary team and referred to specialized centers staffed by experienced and skilled oncology surgeons.

[5] Shibata D, Paty PB, Guillem JG, Wong WD, Cohen AM (2002) Surgical management of isolated retroperitoneal recurrences of colorectal carcinoma. Dis Colon Rectum 45: 795-801.

[6] Dumont F, Kothodinis K, Goe're' D et al (2012) Central retroperitoneal recurrences from colorectal cancer: are lymph node and locoregional recurrences the same disease? Eur J Surg Oncol 38:611-616. 